\title{
THE EFFECT OF BREEDS AND FEED RESTRICTION LEVEL ON SERUM BIOCHEMICAL RESPONSE OF RABBITS
}

\author{
S.M.A. Sallam ${ }^{1}$, S. Ahmed ${ }^{2}$, Omnia Essa ${ }^{2}$ and T. Rabie ${ }^{2}$ \\ 1- Department of Animal and Fish Production, Faculty of Agriculture, (El-Shatby) Alexandria University, 2- \\ Department of Animal Production and Fish Resources, Faculty of Agriculture, Suez Canal University, \\ 41522, Ismailia, Egypt
}

Received: 15/9/2019 Accepted: 6/11/2019

\section{SUMMARY}

The aim of this study was to estimate the rabbit' breed effect (Red Baladi (RB), and New Zealand White $(N Z W)$ breeds), fed ad libitum for the first 11weeks of age then were shifted to the restriction feed for two weeks starting at $11^{\text {th }}$ and $12^{\text {nd }}$ weeks of age, till $15^{\text {th }}$ week of age on body weight gain, mortality rate, and serum biochemical phenotypes. Rabbits were allocated into three groups; control, 60\%, and 80\% from ad libitum feeding to the restricted feed trial implement. Growth performance traits and serum biochemical indices were measured weekly.

During the feed restricted period, the body weight gain was negatively affected in restriction feed groups compared to the control group. However, the average weekly weight gain and feed intake or the feed conversion ratio $(F C R)$ were affected $(P \leq 0.05)$ by either feed restriction or breed over the entire experimental period. Mortality rate (\%) was entirely zero for all studied groups. Although the feed restriction had reduced $(P \leq 0.05)$ the total protein ( $\mathrm{g} / \mathrm{dl})$, triglycerides, $(\mathrm{mg} / \mathrm{dl})$, LDL cholesterol $(\mathrm{mg} / \mathrm{dl})$ and ALT (U/L), AST (U/L) enzymes, but $A L P(U / L)$ and total cholesterol $(\mathrm{mg} / \mathrm{dl})$ did not affect after two weeks of feeding. In conclusion, restriction feeding for growing rabbits starting from $11^{\text {th }}$ weeks of age for two weeks is recommended to improve the serum biochemical indices and therefore the growth performance.

Keywords: feed restriction, growth performance and serum biochemical

\section{INTRODUCTION}

Rabbit meat is an important source of animal protein in Egypt, it provides source of protein while featuring low fat cholesterol content and high quality biological value (Aduku and Olukosi, 1990). Thus, if selected for high feed intake and growth rate, rabbits will provide delicacy and healthy food (Dallezotte, 2000) at reasonable price. Therefore, growing rabbits are usually fed ad libitum to achieve the highest performance of growth rate (Maertens, 2010). However, under certain circumvent areas, feed restriction is used post-weaning to avoid high daily changes in feed intake and the associated digestive disorders (Gidenne et al., 2012; Maertens and Gidenne, 2016) that lead to animal losses. Nevertheless, feed restriction is used also at different intervals at ages of the rabbit life at different levels (Di Meo et al., 2007). Though, feed restriction reducing the growth during the restriction interval, but the growth reduction can be compensated after feed restriction period (Govaerts et al., 2000), so that the high level of feed restriction has the same effect on final live body weight as lower level (Anderson et al., 2005). Furthermore, a positive effect on feed efficiency of restricted animals commenced growth was reported by Tumová et al. (2002); DalleZotte et al. (2005) and De Oliveira et al. (2012), where the feed conversion rate and digestive process for feed were improved (Tůmová et al.,2002; Di Meo et al., 2007 and Abdel-Wareth et al.,2015).Wherever, the compensatory growth depends on the interval and level of restriction (Di Meo et al., 2007; Gidenne et al., 2009a; Romero et al., 2010 and Abdel-Wareth et al., 2015). Feed restriction for a short period of time also changes the nutritional status of system and thus influence blood metabolites concentrations as reported by Renaville et al. (2000); Cabaraux et al. (2003); Guyton and Hall, (2006). Moreover blood characteristics in rabbits are used for infectious detecting stress conditions, and evaluating the metabolic condition of animals (Mohammadalipour et al., 2017). Biochemical blood parameters are influenced by a lot factors including breed (Martinec et al., 2012), and nutrition (Addass et al., 2012; Etim et al., 2014). Furthermore, Van Harten and Cardoso (2010), stated a low concentration of serum biochemical components after feed restriction interval. Therefore, the aim of the current study was to evaluate the effects of levels of feed restriction $(0 \%, 60 \%$ and $80 \%$ from ad libitum intake) for two weeks on growth performance and serum biochemical parameters in Red Baladi and New Zealand white.

\section{MATERIALS AND METHODS}

The present experiment was carried out at the Rabbits Research Farm, Department of Animal Production, Faculty of Agriculture, Suez Canal University, Ismailia, Egypt during the period from September 2017 to February2018.

\section{Experimental design and rabbit management:}

A total of 156 rabbits (78 Red Baladi and 78 New Zealand White) were used in this study. Rabbits were weaned at an age of 28 day and were allocated into 
three groups; each of 26 rabbits. Animals were kept individually in galvanized wire batteries equipped with automatic nipple drinkers and separate feeders. Lighting system was sixteen hr light/eight hr dark in the rabbit during experimental period and apparently healthy and free of any external parasites or skin diseases.Pre-experiment was conducted to carry out the amount of ad libitum feed to calculate the feed restriction levels upon the total daily feed intake. The feed restriction started at $11^{\text {th }}$ wk for two weeks ( $a d$ libitum $0 \%, 60 \%$ and $80 \%$ from ad libitum fed $125 \mathrm{~g}$ ). At the beginning of the $13^{\text {th }}$ week of age, treated groups were fed ad libitum continuously till the slaughter at $15^{\text {th }}$ weeks of age. The calculated chemical components of the diet were $18 \%$ crude protein, $2.69 \%$ fat, $12.39 \%$ crude fiber and $2738 \mathrm{kcal}$ digestible energy/kg diet. The rabbits were fed according to NRC requirement (1984).

\section{Performance and Serum biochemical parameters Growth traits:}

Rabbits were individually weighed weekly at fixed day early in the morning before feeding. Then the body weight gain, feed intake $(\mathrm{g})$, FCR $(\mathrm{kg}$ feed/kg gain) and mortality rate was recoded.

European Efficiency factors (EEf) were calculated according to Nilipour, (1998):

$\mathrm{EEF}=($ weight gain $(\mathrm{g}) /$ age day $) \times($ viability rate \%/ FCR (kg feed/kg gain): 10 .

\section{Serum biochemical parameters:}

Three blood samples were withdrawn from the ear vein of individual rabbits during $11^{\text {th }}, 12^{\text {th }}$ weeks and at the slaughter $\left(15^{\text {th }}\right.$ week of age $)$. At the end of the experiment $\left(15^{\text {th }}\right.$ week of age), rabbits treatments within breed were individually weighted after $12 \mathrm{~h}$ fasting slaughtered and bleed for 3-5 minutes,

Serum was separated by centrifugation of the blood samples at $3000 \mathrm{rpm}$ for 15 minutes and stored at $-20^{\circ} \mathrm{C}$ until analysis. Total protein, total cholesterol, triglycerides, LDL cholesterol, HDL cholesterol and liver enzymes activity (alanine aminotransferase (ALT), aspartate aminotransferase (AST) and aspartate aminotransferase (ALP) were calorimetrically determined using commercial kits (Reactivos GPL CHEMELEX, S.A Pol. Ind. Can Castells. C / Industria 113, Nau J 08420 Canovelles Barcelona), as described by Young and Friedman, (2001).

\section{Statistical analysis:}

Data were statistically analyzed using General Linear Models Procedure of the SPSS 20 program (2011).

A $3 \times 2$ factorial design was used. The following model was used to study the effect of main factors and interaction between feed restriction levels (FR) and Breed $(\mathrm{B})$ on parameters investigated as follows: $\mathrm{Y}_{\mathrm{ijk}}=\mathrm{u}+\mathrm{T}_{\mathrm{i}}+\mathrm{B}_{\mathrm{j}}+(\mathrm{TB})_{\mathrm{ij}}+\mathrm{e}_{\mathrm{ijk}}$

Where:

$Y_{\mathrm{ijk}}=$ An observation on mention off analyzed traits $\mathrm{u}=$ overall mean $; \mathrm{T}_{\mathrm{i}}=$ effect of $\mathrm{FR}$ level; $\mathrm{I}=(1,2$ and 3 ); $B_{j}=$ effect of breed; ${ }_{j}=(1$ and 2$) ;(T B)_{i j}=$ effect of interaction between FR and B ( ${ }_{i j}(1,2 \ldots .6)$; and $e_{j i k}=$ Experimental error. The Differences means among treatments were subjected to Duncan' $\mathrm{s}$ Multiple Range- test (Duncan, 1955).

\section{RESULTS AND DISCUSSION}

\section{Growth Performance: \\ Weight gain:}

The effect of breed and feed restriction levels and their interaction effects on weight gain atthe $1^{\text {st }}$ week, $2^{\text {nd }}$ weeks and after feed restriction period are presented in a Table (1). The feed restriction levels had negative effects $(\mathrm{P} \leq 0.05)$ on weight gain (g) in two weeks of feed restriction, but switched to positive effect $(\mathrm{P} \leq 0.05)$ after feed restriction period. There was also interaction effect between breed and the levels of feed restriction on weight gain $(\mathrm{P} \leq 0.05)$. At the beginning of the feed restriction period, the feed intake was reduced and backed to normal a $d$ libitum intake to compensate rabbits growth, which is in agreement with Birolo et al., (2017) and Tůmová et al. (2018).

\section{Feed efficiency traits:}

The feed intake was lower $(\mathrm{P}<0.05)$ in the $60 \%$ restriction feeding group (FR1) than the d-libitum group (FR0) $(\mathrm{P}<0.05)$ but the total feed intake of rabbits was not significantly $(\mathrm{P}>0.05)$ changed between the two breeds, which may be due to equality total feed intake among treatments. Moreover, FCR ( $\mathrm{kg}$ feed $/ \mathrm{kg}$ gain) and (EEf) were significantly lower $(\mathrm{P}<0.05)$ in the $(\mathrm{FR} 0)$ than in the (FR1) group $(\mathrm{P}<0.05)$, with no breed difference detected $(\mathrm{P}<0.05)$ as shown in Table (2). The feed intake was similar for control and feed restricted groups, which might be related to feeding behavior contributes to the results of feed intake (Tumova et al., 2003); Romero et al., 2010; Gidenne et al., 2012). The improvement of FCR might be associated with the morphological and physiological changes in the intestine in the restricted rabbits, which had a longer small intestine (Oliveira et al., 2012) and a longer retention time. In contrary, Gidenne et al. (2009b) found that the compensatory growth was not linked with evolved feed conversion in which, where feed convers was increased in restricted feed groups compared to the ad libitum group.

The results revealed that the feed restriction had no significant effect on mortality rate (\%) and similar results were reported by Foubert et al. (2008); Ebeid et al. (2012). While along restriction period for 2 or 3 weeks to decrease mortality rate and digestive problems (Gidenne et al., 2012). Morover,the duration of feed restriction had no influence on mortality percentage as reported by Gidenne et al. (2003); Yakubu et al. (2007). 
Table 1. Effect of breed, feed restriction and their interaction on weight gain (g) of rabbits at different age out the experiment

\begin{tabular}{|c|c|c|c|c|c|c|c|c|c|c|c|}
\hline \multirow[t]{2}{*}{ Weeks } & \multicolumn{2}{|c|}{ Breed } & \multicolumn{3}{|c|}{ FR } & \multicolumn{6}{|c|}{ Inter. Effects: B*FR } \\
\hline & RB & NZW & FR0 & FR1 & FR2 & RB*FR0 & RB*FR1 & RB*FR2 & NZW*FR0 & NZW*FR1 & NZW*FR2 \\
\hline \multirow[t]{2}{*}{$1^{\mathrm{st}} \mathrm{wk}(\mathrm{FR})$} & $97.1^{b}$ & $105.1^{\mathrm{a}}$ & $200.1^{\mathrm{a}}$ & $72 . .^{4 a b}$ & $111.3^{b}$ & $195.3^{\mathrm{a}}$ & $66.9^{\mathrm{ab}}$ & $80.1^{\mathrm{b}}$ & $203.2^{\mathrm{a}}$ & $70.0^{\mathrm{ab}}$ & $105.1^{b}$ \\
\hline & \pm 4.99 & \pm 5.01 & \pm 2.79 & \pm 3.89 & \pm 3.61 & \pm 5.82 & \pm 5.82 & \pm 5.82 & \pm 5.79 & \pm 5.79 & \pm 5.79 \\
\hline \multirow[t]{2}{*}{$2^{\text {nd }}$ wk (FR) } & $61.6^{\mathrm{b}}$ & $73.8^{\mathrm{a}}$ & $201.8^{\mathrm{a}}$ & $-7.92^{\mathrm{c}}$ & $9.3^{b}$ & $201.3^{\mathrm{a}}$ & $-19.3^{c}$ & $2.79^{b}$ & $202.2^{\mathrm{a}}$ & $3.2^{\mathrm{c}}$ & $15.8^{\mathrm{b}}$ \\
\hline & \pm 3.30 & \pm 3.30 & \pm 4.04 & \pm 4.10 & \pm 3.995 & \pm 5.74 & \pm 5.68 & \pm 5.74 & \pm 5.71 & \pm .5 .68 & \pm 5.71 \\
\hline \multirow[t]{2}{*}{ After (FR) } & $328.8^{\mathrm{b}}$ & $332.4^{\mathrm{a}}$ & $200.0^{\mathrm{c}}$ & $404.6^{\mathrm{a}}$ & $387.3^{b}$ & $201.1^{\mathrm{c}}$ & 404.1 & $381.2^{\mathrm{b}}$ & $198.9^{c}$ & 405.1 & $393.5^{b}$ \\
\hline & \pm 3.96 & \pm 3.96 & \pm 4.85 & \pm 4.90 & \pm 4.80 & \pm 6.89 & ${ }^{\mathrm{a}} \pm 6.82$ & \pm 6.90 & \pm 6.86 & ${ }^{\mathrm{a}} \pm 6.82$ & \pm 6.86 \\
\hline \multirow[t]{2}{*}{ Atslaughter } & $196.6^{\mathrm{b}}$ & $289.3^{\mathrm{a}}$ & $229 .^{2 a b}$ & $249.9^{\mathrm{a}}$ & $249.2^{b}$ & $170.8^{\mathrm{ab}}$ & 200.4 & 218.8 & $287.5^{\mathrm{b}}$ & 299.3 & $280.4^{\mathrm{ab}}$ \\
\hline & \pm 6.03 & \pm 6.03 & \pm 7.38 & \pm 7.31 & \pm 7.46 & \pm 10.50 & ${ }^{\mathrm{a}} \pm 10.39$ & $\mathrm{~b}_{ \pm} \pm 10.50$ & \pm 10.44 & ${ }^{\mathrm{a}} \pm 10.39$ & \pm 10.44 \\
\hline
\end{tabular}

First wk (FR): Frist week form feed restriction; $2^{\text {nd }} w k(F R)$ : two week form feed restriction RB: Red Baladi Breed; NZW: New Zealand White Breed; FR0: control diet; FR1:60\% of the diet; FR2:80\% of the Diet;B *FR: Interaction between breed and feed; \pm : standard error of the mean;a-b Means within the same row with the different superscript letter are significantly different $(\mathrm{p}>.05)$.

Table 2. Interaction effects on feed intake (g), feed conversion ratio ( $\mathrm{kg} \mathrm{feed} / \mathrm{kg}$ gain) and economic efficiency factors at slaughter age

\begin{tabular}{lcccccc}
\hline Traits & \multicolumn{7}{c}{ Inter. Effects: B*FR } \\
\cline { 2 - 7 } & RB*FR0 & RB*FR1 & RB*FR2 & NZW*FR0 & NZW*FR1 & NZW $^{*}$ FR2 \\
\hline TFI & $7175^{\mathrm{a}}$ & $6895^{\mathrm{b}}$ & $6615 \mathrm{a}^{\mathrm{b}}$ & $7175^{\mathrm{a}}$ & $6895^{\mathrm{b}}$ & $6615^{\mathrm{ab}}$ \\
FCR & $3.37^{\mathrm{a}}$ & $3.07^{\mathrm{b}}$ & $2.82^{\mathrm{ab}}$ & $3.04^{\mathrm{a}}$ & $2.82^{\mathrm{b}}$ & $2.88^{\mathrm{ab}}$ \\
EEf & $5.9^{\mathrm{ab}}$ & $6.7^{\mathrm{b}}$ & $7.3^{\mathrm{a}}$ & $7.2^{\mathrm{ab}}$ & $8.3^{\mathrm{a}}$ & $8.1^{\mathrm{b}}$ \\
\hline
\end{tabular}

B*FR: Interaction between breed and feed; T.F.I: total feed intake (g) from (4) weeks to slaughter; FCR: Feed conversion ratio (kg feed/kg gain) at slaughter; (EEf):Economic Efficiency factor

Serum biochemical parameters:

The values of all serum biochemical characteristics in the current study were within the physiological range as described by Hewitt et al. (1989); Archetti et al. (2008). The effect of breed, feed restriction levels and their interaction effects on the total protein $(\mathrm{g} / \mathrm{dl})$,total cholesterol (mg/dl), triglycerides (mg/dl),LDL cholesterol (mg/dl) and HDL cholesterol (mg/dl), at $1^{\text {st }}$ week, $2^{\text {nd }}$ week and after feed restriction are shown in Tables $(3 \& 4)$. Breed, feed restriction and their interaction affects $(\mathrm{P}<0.05)$ at the $1^{\text {st }}$ week, $2^{\text {nd }}$ week and after feed restriction reduced serum total protein. Feed restriction had reduced $(\mathrm{P} \leq$ $0.05)$ total protein as stated with Rajman et al. (2006); Van Harten and Cardoso (2010); ElSpeiy et al. (2015), where Archetti et al. (2008) and Özkan et al., (2012) explain that the concentrations of total protein are related to protein metabolism. The total cholesterol $(\mathrm{mg} / \mathrm{dl})$ had no effect $(\mathrm{P} \leq 0.05)$ after $2^{\text {nd }}$ week by feed restriction, but it had reduced $(\mathrm{P} \leq 0.05)$ at slaughter time, which in agreement with Beshara et al. (2017).In contrast, El-Speiy et al. (2015) showed that total cholesterol concentration increased at the end of the rabbits treated with feed restriction. The triglycerides $(\mathrm{mg} / \mathrm{dl})$ was significantly $(\mathrm{P} \leq 0.05)$ reduced due to feed restriction, which was in agreement with Van Harten and Cardoso, (2010) and El-Speiy et al. (2015). In connection with the feed restriction had positive effect $(\mathrm{P} \leq 0.05)$ on $\mathrm{LDL}$ and HDL cholesterol $(\mathrm{mg} / \mathrm{dl})$ identical to Van Harten and Cardoso, (2010); Fatma and Hayam, (2014) and Darina et al. (2018).

Table 3. Effect of breed, feed restriction and their interaction on total protein (g/d) and total cholesterol (mg/dl)

\begin{tabular}{|c|c|c|c|c|c|c|c|c|c|c|c|}
\hline \multirow[t]{2}{*}{ Traits } & \multicolumn{2}{|c|}{ Breed } & \multicolumn{3}{|c|}{ FR } & \multicolumn{6}{|c|}{ Inter. Effects: B*FR } \\
\hline & RB & NZW & FR0 & FR1 & FR2 & RB*FR0 & RB*FR1 & RB*FR2 & NZW*FR0 & NZW*FR1 & NZW*FR2 \\
\hline \multicolumn{12}{|c|}{ Total protein $(\mathrm{g} / \mathrm{dl})$} \\
\hline $\begin{array}{l}11^{\text {th }} \text { week } \\
(\mathrm{FR})\end{array}$ & $\begin{array}{c}6.02^{\mathrm{a}} \\
\pm 0.028\end{array}$ & $\begin{array}{c}5.43^{\mathrm{b}} \\
\pm 0.091\end{array}$ & $\begin{array}{c}5.69^{\mathrm{b}} \\
\pm 0.058\end{array}$ & $\begin{array}{c}5.85^{\mathrm{a}} \\
\pm 0.054\end{array}$ & $\begin{array}{c}5.64^{\mathrm{ab}} \\
\pm 0.029\end{array}$ & $\begin{array}{l}6.01^{b} \\
\pm 0.04\end{array}$ & $\begin{array}{c}6.21^{\mathrm{a}} \\
\pm 0.048\end{array}$ & $\begin{array}{c}5.85^{\mathrm{ab}} \\
\pm 0.044\end{array}$ & $\begin{array}{c}5.36^{\mathrm{ab}} \\
\pm 0.052\end{array}$ & $\begin{array}{c}5.49^{\mathrm{a}} \\
\pm 0.028\end{array}$ & $\begin{array}{c}5.42^{\mathrm{b}} \\
\pm 0.031\end{array}$ \\
\hline $\begin{array}{l}12^{\text {th }} \text { week } \\
\text { (FR) } \\
\text { Atslaughter }\end{array}$ & $\begin{array}{c}5.77^{\mathrm{a}} \\
\pm 0.046 \\
6.16^{\mathrm{a}} \\
\pm 0.042\end{array}$ & $\begin{array}{c}5.36^{\mathrm{b}} \\
\pm 0.035 \\
5.83^{\mathrm{b}} \\
\pm 0.037\end{array}$ & $\begin{array}{c}5.93^{\mathrm{a}} \\
\pm 0.052 \\
6.28^{\mathrm{a}} \\
\pm 0.054\end{array}$ & $\begin{array}{c}5.32^{\mathrm{ab}} \\
\pm 0.034 \\
5.87^{\mathrm{b}} \\
\pm 0.039\end{array}$ & $\begin{array}{c}5.44^{\mathrm{b}} \\
\pm 0.042 \\
5.83^{\mathrm{ab}} \\
\pm 0.041\end{array}$ & $\begin{array}{c}6.24^{\mathrm{a}} \\
\pm 0.043 \\
6.60^{\mathrm{a}} \\
\pm 0.054\end{array}$ & $\begin{array}{c}5.51^{\mathrm{b}} \\
\pm 0.042 \\
5.96^{\mathrm{b}} \\
\pm 0.034\end{array}$ & $\begin{array}{c}5.55^{\mathrm{b}} \\
\pm 0.041 \\
5.91^{\mathrm{b}} \\
\pm 0.039\end{array}$ & $\begin{array}{c}5.63^{\mathrm{a}} \\
\pm 0.036 \\
5.96 \\
{ }^{\mathrm{a}} \pm 0.029\end{array}$ & $\begin{array}{c}5.13^{\mathrm{ab}} \\
\pm 0.072 \\
5.77^{\mathrm{b}} \\
\pm 0.021\end{array}$ & $\begin{array}{c}5.34^{\mathrm{b}} \\
\pm 0.078 \\
5.76^{\mathrm{b}} \\
\pm 0.076\end{array}$ \\
\hline \multicolumn{12}{|c|}{ Total cholesterol(mg/dl) } \\
\hline $11^{\text {th }}$ week & $84.13^{\mathrm{a}}$ & $82.99^{b}$ & $82.02^{\mathrm{ab}}$ & $84.67^{\mathrm{a}}$ & $83.98^{b}$ & $82.29^{\mathrm{a}}$ & $85.96^{\mathrm{a}}$ & $84.13^{\mathrm{a}}$ & $81.75^{\mathrm{a}}$ & $83.44^{\mathrm{a}}$ & $83.83^{\mathrm{a}}$ \\
\hline (FR) & \pm 0.258 & \pm 0.258 & \pm 0.316 & \pm 0.313 & \pm 0.320 & \pm 0.450 & \pm 0.445 & \pm 0.450 & \pm 0.447 & \pm 0.445 & \pm 0.447 \\
\hline $12^{\text {th }}$ week & $83.61^{\mathrm{a}}$ & $82.20^{\mathrm{b}}$ & $82.92^{\mathrm{a}}$ & $82.71^{\mathrm{a}}$ & $83.09^{\mathrm{a}}$ & $83.29^{\mathrm{a}}$ & $84.08^{\mathrm{a}}$ & $83.46^{\mathrm{a}}$ & $82.54^{\mathrm{a}}$ & $81.36^{\mathrm{a}}$ & $82.74^{\mathrm{a}}$ \\
\hline (FR) & \pm 0.55 & \pm 0.55 & \pm 0.313 & \pm 0.309 & \pm 0.316 & \pm 0.444 & \pm 0.444 & \pm 0.440 & \pm 0.442 & \pm 0.442 & \pm 0.440 \\
\hline Atslaughter & $83.26^{\mathrm{a}}$ & $82.35^{\mathrm{b}}$ & $84.29^{\mathrm{a}}$ & $82.34^{\mathrm{b}}$ & $81.79^{\mathrm{ab}}$ & $86.63^{\mathrm{a}}$ & $83.42^{b}$ & $79.75^{\mathrm{ab}}$ & $81.96^{\mathrm{b}}$ & $81.28^{\mathrm{ab}}$ & $83.96^{\mathrm{a}}$ \\
\hline & \pm 0.343 & \pm 0.343 & \pm 0.420 & \pm 0.416 & \pm 0.425 & \pm 0.597 & \pm 0.591 & \pm 0.594 & \pm 0.597 & \pm 0.591 & \pm 0.594 \\
\hline
\end{tabular}

First wk (FR): First week form feed restriction; $2^{\text {nd }}$ wk (FR): two week form feed restriction; RB: Red Baladi Breed; NZW: New Zealand White Breed; FR0: control diet; FR1:60\% of the diet; FR2:80\% of the Diet; B *FR: Interaction between breed and feed; \pm : standard error of the mean; a-b Means within the same row with the different superscript letter are significantly different (p>.05). 
Table 4. Effect of breed, feed restriction and their interaction on triglycerides, LDL cholesterol and HDL cholesterol

\begin{tabular}{|c|c|c|c|c|c|c|c|c|c|c|c|}
\hline \multirow[t]{2}{*}{ Traits } & \multicolumn{2}{|c|}{ Breed } & \multicolumn{3}{|c|}{ FR } & \multicolumn{6}{|c|}{ Inter. Effects: B*FR } \\
\hline & RB & NZW & FR0 & FR1 & FR2 & RB*FR0 & RB*FR1 & RB*FR2 & NZW*FR0 & NZW*FR1 & NZW*FR2 \\
\hline \multicolumn{12}{|c|}{ Triglycerides $(\mathrm{mg} / \mathrm{dl})$} \\
\hline $11^{\text {th }}$ & $85.22^{\mathrm{a}}$ & $74.49^{b}$ & $79.79^{a}$ & $80.02^{\mathrm{a}}$ & $79.74^{\mathrm{a}}$ & $84.79^{\mathrm{a}}$ & $85.96^{\mathrm{a}}$ & $84.92^{\mathrm{a}}$ & $74.79^{\mathrm{a}}$ & $74.32^{\mathrm{a}}$ & $74.35^{\mathrm{a}}$ \\
\hline $\begin{array}{l}\text { week } \\
\text { (FR) }\end{array}$ & \pm 0.217 & \pm 0.217 & \pm 0.266 & \pm 0.263 & \pm 0.268 & \pm 0.378 & \pm 0.378 & \pm 0.374 & \pm 0.376 & \pm 0.376 & \pm 0.374 \\
\hline $12^{\text {th }}$ & $85.08^{a}$ & $71.72^{b}$ & $80.04^{\mathrm{a}}$ & $76.59^{\mathrm{ab}}$ & $78.62^{b}$ & $85.42^{\mathrm{a}}$ & $85.42^{\mathrm{a}}$ & $84.42^{b}$ & $74.67^{\mathrm{a}}$ & $68.12^{\mathrm{ab}}$ & $72.57^{\mathrm{b}}$ \\
\hline $\begin{array}{l}\text { week } \\
\text { (FR) }\end{array}$ & \pm 0.287 & \pm 0.287 & \pm 0.352 & \pm 0.348 & \pm 0.355 & \pm 0.500 & \pm 0.495 & \pm 0.500 & \pm 0.495 & \pm 0.489 & \pm 0.498 \\
\hline At & $85.43^{a}$ & $71.36^{\mathrm{b}}$ & $80.13^{a}$ & $76.59^{\mathrm{ab}}$ & $78.51^{b}$ & $86.46^{\mathrm{a}}$ & 85.42 & $84.42^{\mathrm{ab}}$ & $73.79^{\mathrm{a}}$ & $68.12^{\mathrm{ab}}$ & $72.35^{b}$ \\
\hline slaughter & \pm 0.266 & \pm 0.266 & \pm 0.335 & \pm 0.329 & \pm 0.322 & \pm 0.463 & $\mathrm{~b}_{ \pm 0.485}$ & \pm 0.463 & \pm 0.460 & \pm 0.458 & \pm 0.460 \\
\hline \multicolumn{12}{|c|}{ LDL cholesterol $(\mathrm{mg} / \mathrm{dl})$} \\
\hline $11^{\text {th }}$ & $32.58^{\mathrm{a}}$ & $32.83^{\mathrm{a}}$ & $31.81^{\mathrm{ab}}$ & $33.41^{\mathrm{a}}$ & $32.89^{b}$ & $31.33^{\mathrm{a}}$ & $33.67^{\mathrm{a}}$ & $32.75^{\mathrm{a}}$ & $32.29^{\mathrm{a}}$ & $33.16^{\mathrm{a}}$ & $33.04^{\mathrm{a}}$ \\
\hline $\begin{array}{l}\text { week } \\
\text { (FR) }\end{array}$ & \pm 0.149 & \pm 0.149 & \pm 0.182 & \pm 0.181 & \pm 0.184 & \pm 0.259 & \pm 0.257 & \pm 0.259 & \pm 0.258 & \pm 0.257 & \pm 0.258 \\
\hline $12^{\text {th }}$ & $29.40^{\mathrm{a}}$ & $29.98^{\mathrm{a}}$ & $31.85^{\mathrm{a}}$ & $28.19^{\mathrm{ab}}$ & $29.02^{b}$ & $31.33^{\mathrm{a}}$ & $28.42^{\mathrm{ab}}$ & $28.46^{\mathrm{b}}$ & $32.38^{\mathrm{a}}$ & $28.00^{\mathrm{ab}}$ & $29.61^{b}$ \\
\hline $\begin{array}{l}\text { week } \\
\text { (FR) }\end{array}$ & \pm 0.150 & \pm 0.150 & \pm 0.184 & \pm 0.182 & \pm 0.186 & \pm 0.261 & \pm 0.260 & \pm 0.258 & \pm 0.260 & \pm 0.260 & \pm 0.258 \\
\hline & $27.57^{b}$ & $28.43^{\mathrm{a}}$ & $32.19^{\mathrm{a}}$ & $25.48^{\mathrm{ab}}$ & $26.33^{b}$ & $31.96^{\mathrm{a}}$ & $26.04^{\mathrm{b}}$ & $24.71^{\mathrm{ab}}$ & $32.42^{\mathrm{a}} \pm 0.367$ & $24.96^{\mathrm{ab}}$ & $28.00^{\mathrm{b}}$ \\
\hline slaughter & \pm 0.212 & \pm 0.212 & \pm 0.260 & \pm 0.257 & \pm 0.262 & \pm 0.369 & \pm 0.365 & \pm 0.369 & & \pm 0.365 & \pm 0.367 \\
\hline \multicolumn{12}{|c|}{ HDL cholesterol $(\mathrm{mg} / \mathrm{dl})$} \\
\hline $11^{\text {th }}$ & $33.55^{b}$ & 34.87 & $33.58^{\mathrm{ab}}$ & 34.76 & $34.29^{b}$ & 32.96 & 34.00 & 33.71 & 34.21 & $35.52^{\mathrm{a}}$ & $34.87^{\mathrm{a}}$ \\
\hline $\begin{array}{l}\text { week } \\
\text { (FR) }\end{array}$ & \pm 0.151 & ${ }^{\mathrm{a}} \pm 0.151$ & \pm 0.185 & ${ }^{\mathrm{a}} \pm 0.183$ & \pm 0.183 & ${ }^{\mathrm{a}} \pm 0.263$ & ${ }^{\mathrm{a}} \pm 0.260$ & ${ }^{\mathrm{a}} \pm 0.263$ & ${ }^{\mathrm{a}} \pm 0.263$ & \pm 0.261 & \pm 0.260 \\
\hline $12^{\text {th }}$ & $35.83^{b}$ & 37.37 & $34.71^{\mathrm{ab}}$ & 38.21 & $36.89^{b}$ & $34.17^{\mathrm{ab}}$ & 36.79 & $36.54^{\mathrm{b}}$ & $35.25^{\mathrm{ab}}$ & $39.60^{\mathrm{a}}$ & $37.22^{b}$ \\
\hline $\begin{array}{l}\text { week } \\
\text { (FR) }\end{array}$ & \pm 0.166 & ${ }^{\mathrm{a}} \pm 0.166$ & \pm 0.204 & ${ }^{\mathrm{a}} \pm 0.202$ & \pm 0.206 & \pm 0.289 & ${ }^{\mathrm{a}} \pm 0.286$ & \pm 0.288 & \pm 0.288 & \pm 0.286 & \pm 0.288 \\
\hline & $37.78^{\mathrm{b}}$ & $39.75^{\mathrm{a}}$ & $35.65^{\mathrm{ab}}$ & $41.75^{\mathrm{a}}$ & $38.89^{\mathrm{b}}$ & $35.08^{\mathrm{a}}$ & $40.71^{\mathrm{a}}$ & $37.54^{\mathrm{a}}$ & $36.21^{\mathrm{a}}$ & $42.80^{\mathrm{a}}$ & $40.26^{\mathrm{a}}$ \\
\hline slaughter & \pm 0.222 & \pm 0.222 & \pm 0.272 & \pm 0.270 & \pm 0.275 & \pm 0.387 & \pm 0.383 & \pm 0.387 & \pm 0.385 & \pm 0.385 & \pm 0.383 \\
\hline
\end{tabular}

Table 5. Effect of breed, feed restriction and their interaction on ALT,AST and ALP (U/L)enzymes

\begin{tabular}{|c|c|c|c|c|c|c|c|c|c|c|c|}
\hline \multirow[t]{2}{*}{ Traits } & \multicolumn{2}{|c|}{ Breed } & \multicolumn{3}{|c|}{ FR } & \multicolumn{6}{|c|}{ Inter. Effects: B*FR } \\
\hline & RB & NZW & FR0 & FR1 & FR2 & RB*FR0 & RB*FR1 & RB*FR2 & NZW*FR0 & NZW*FR1 & NZW*FR2 \\
\hline \multicolumn{12}{|c|}{$A L T(U / L)$} \\
\hline $11^{\text {th }}$ & $46.13^{a}$ & $42.57^{b}$ & $44.06^{\mathrm{a}}$ & $44.59^{a}$ & 44.39 & $45.67^{\mathrm{a}}$ & 46.38 & 46.33 & $42.46^{\mathrm{a}}$ & 42.80 & $42.43^{\mathrm{a}}$ \\
\hline $\begin{array}{l}\text { week } \\
\text { (FR) }\end{array}$ & \pm 0.124 & \pm 0.124 & \pm 0.152 & \pm 0.150 & ${ }^{\mathrm{a}} \pm 0.153$ & \pm 0.216 & ${ }^{\mathrm{a}} \pm 0.213$ & ${ }^{\mathrm{a}} \pm 0.216$ & \pm 0.213 & ${ }^{\mathrm{a}} \pm 0.213$ & \pm 0.215 \\
\hline $12^{\text {th }}$ & 44.44 & $39.60^{\mathrm{b}}$ & 45.67 & $39.51^{\mathrm{ab}}$ & $40.89^{b}$ & $46.46^{\mathrm{a}}$ & $43.08^{\mathrm{ab}}$ & $43.79^{\mathrm{b}}$ & $44.87^{\mathrm{a}}$ & $36.00^{\mathrm{ab}}$ & $37.96^{\mathrm{b}}$ \\
\hline $\begin{array}{l}\text { week } \\
\text { (FR) }\end{array}$ & ${ }^{\mathrm{a}} \pm 0.220$ & \pm 0.220 & ${ }^{\mathrm{a}} \pm 0.269$ & \pm 0.267 & \pm 0.272 & \pm 0.383 & \pm 0.379 & \pm 0.383 & \pm 0.381 & \pm 0.379 & \pm 0.381 \\
\hline At & $47.36^{\mathrm{a}}$ & $42.83^{\mathrm{b}}$ & $47.35^{\mathrm{a}}$ & $43.68^{\mathrm{ab}}$ & $44.29^{\mathrm{b}}$ & $47.96^{\mathrm{a}}$ & $46.50^{\mathrm{ab}}$ & $47.62^{\mathrm{b}}$ & $46.75^{\mathrm{a}}$ & $40.88^{\mathrm{ab}}$ & $40.91^{\mathrm{b}}$ \\
\hline slaughter & \pm 0.235 & \pm 0.235 & \pm 0.288 & \pm 0.285 & \pm 0.291 & \pm 0.410 & \pm 0.406 & \pm 0.410 & \pm 0.408 & \pm 0.406 & \pm 0.408 \\
\hline \multicolumn{12}{|c|}{$A S T(U / L)$} \\
\hline $11^{\text {th }}$ & $37.06^{b}$ & 41.12 & 38.98 & 39.26 & 39.02 & $37.37^{\mathrm{a}}$ & $36.46^{\mathrm{ab}}$ & $37.33^{b}$ & $40.58^{\mathrm{ab}}$ & $42.04^{\mathrm{a}}$ & $40.70^{b}$ \\
\hline $\begin{array}{l}\text { week } \\
\text { (FR) }\end{array}$ & \pm 0.126 & ${ }^{\mathrm{a}} \pm 0.126$ & ${ }^{\mathrm{a}} \pm 0.154$ & ${ }^{a} \pm 0.152$ & ${ }^{\mathrm{a}} \pm 0.156$ & \pm 0.219 & \pm 0.217 & \pm 0.219 & \pm 0.218 & \pm 0.217 & \pm 0.218 \\
\hline $12^{\text {th }}$ & $35.08^{b}$ & 37.83 & $39.42^{\mathrm{a}}$ & $33.63^{\mathrm{ab}}$ & $36.33^{b}$ & 38.25 & $32.67^{\mathrm{ab}}$ & $34.33^{b}$ & $40.58^{\mathrm{a}}$ & $34.60^{\mathrm{ab}}$ & $38.35^{\mathrm{b}}$ \\
\hline $\begin{array}{l}\text { week } \\
\text { (FR) }\end{array}$ & \pm 0.162 & ${ }^{\mathrm{a}} \pm 0.162$ & \pm 0.198 & \pm 0.196 & \pm 0.200 & ${ }^{\mathrm{a}} \pm 0.281$ & \pm 0.278 & \pm 0.278 & \pm 0.280 & \pm 0.281 & \pm 0.278 \\
\hline At & $37.78^{\mathrm{b}}$ & $43.85^{\mathrm{a}}$ & $40.63^{b}$ & $41.55^{\mathrm{a}}$ & $40.28^{\mathrm{ab}}$ & $39.83^{\mathrm{a}} \pm$ & $35.79^{\mathrm{ab}}$ & $37.71^{\mathrm{b}}$ & $41.42^{\mathrm{ab}}$ & $47.20^{\mathrm{a}}$ & $42.83^{\mathrm{b}}$ \\
\hline slaughter & \pm 0.279 & \pm 0.279 & \pm 0.342 & \pm 0.339 & \pm 0.346 & 0.486 & \pm 0.481 & \pm 0.486 & \pm 0.484 & \pm 0.481 & \pm 0.484 \\
\hline \multicolumn{12}{|c|}{$A L P(U / L)$} \\
\hline $11^{\text {th }}$ & $74.92^{b}$ & 82.23 & 78.27 & 78.43 & 79.05 & $74.96^{\mathrm{a}}$ & 74.58 & 75.21 & $81.58^{\mathrm{a}}$ & $82.24^{\mathrm{a}}$ & 82.87 \\
\hline $\begin{array}{l}\text { week } \\
\text { (FR) }\end{array}$ & \pm 0.232 & ${ }^{\mathrm{a}} \pm 0.232$ & ${ }^{\mathrm{a}} \pm 0.284$ & ${ }^{\mathrm{a}} \pm 0.281$ & ${ }^{\mathrm{a}} \pm 0.287$ & \pm 0.404 & ${ }^{\mathrm{a}} \pm 0.400$ & ${ }^{\mathrm{a}} \pm 0.404$ & \pm 0.402 & \pm 0.400 & ${ }^{\mathrm{a}} \pm 0.402$ \\
\hline $12^{\text {th }}$ & $72.07^{b}$ & 79.75 & $78.69^{\mathrm{a}}$ & $73.15^{\mathrm{ab}}$ & $75.89^{b}$ & 74.96 & 69.63 & 71.63 & $82.42^{\mathrm{a}}$ & $76.68^{a}$ & 80.17 \\
\hline $\begin{array}{l}\text { week } \\
\text { (FR) }\end{array}$ & \pm 0.245 & ${ }^{\mathrm{a}} \pm 0.245$ & \pm 0.300 & \pm 0.297 & \pm 0.303 & ${ }^{\mathrm{a}} \pm 0.426$ & ${ }^{\mathrm{a}} \pm 0.421$ & ${ }^{\mathrm{a}} \pm 0.426$ & \pm 0.424 & \pm 0.421 & ${ }^{\mathrm{a}} \pm 0.424$ \\
\hline At & $76.58^{b}$ & $83.25^{\mathrm{a}} \pm$ & $80.06^{\mathrm{a}}$ & $79.57^{\mathrm{a}}$ & $80.13^{\mathrm{a}_{ \pm}}$ & $75.96^{\mathrm{b}}$ & $76.92^{\mathrm{a}}$ & $76.88^{\mathrm{ab}}$ & $84.17^{\mathrm{a}}$ & $82.12^{\mathrm{ab}}$ & $83.52^{\mathrm{b}}$ \\
\hline slaughter & \pm 0.220 & 0.220 & \pm 0.269 & \pm 0.272 & 0.269 & \pm 0.383 & \pm 0.379 & \pm 0.383 & \pm 0.381 & \pm 0.379 & \pm 0.381 \\
\hline
\end{tabular}

The effect of breed, feed restriction levels and interaction effects between breed and feeding on alanine aminotransferase (ALT), aspartate aminotransferase (AST) and alkaline 
phosphatase (ALP) (U/L) enzymes in first week, 2nd week and after feed restriction in table (5).The feed restriction had reduced significantly $(\mathrm{P} \leq 0.05)$ ALT and AST at slaughter time, which is inconsistent with Gallois et al. (2005); Fatma and Hayam, (2014); Beshara et al. (2017) and Darina et al., 2018). In contrast, Amber et al. (2014) and Beshara et al. (2017) reported that the feed restriction did not significantly affect the ALT, AST and ALP.

\section{CONCLUSIONS}

Rabbits to feed restriction for two weeks stared in $11^{\text {th }}$ wk compared with rabbits fed $a d$ libitum up to slaughter time hadn't negative effect on growth performance, mortality and serum biochemical parameters. So, it is interesting to note that feed restriction will reduce feed costs of rabbits.

\section{REFERENCES}

Abdel-Wareth, A.A.A., S. Kehraus, A.H.H. Ali, Z.S.H. Ismail and K.H. Sudekum, 2015. Effects of temporary intensive feed restriction on performance, nutrient digestibility and carcas criteria of growing male Californian rabbits. Arch. Anim. Nutr., 69: 69-78. https://doi.org/10.1080/174503 9X.2014.1002672.

Addass, PA., D.I. David, A.Edward, K.E. Zira and A. Midak, 2012.Effect of age, sex and management system on some haematological parameters of intensively and semiintensively kept chicken in Mubi, Adamawa State, Nigeria. Iranian Journal of Applied Animal Science 2: 277-282.

Aduku, A.O. and O.Olukosi,1990. Rabbit management in the tropics production, processing, utilization, marketing, economic practical training, research and future prospects. Living Book Series, G. U. Publications, Abuja.

Amber, K.H., M.Fatma, Abd El-Nabil, W.A. Morsy, and H.A. Shama, 2014.Effect of dietary supplementation of probiotic and prebiotic on preventing post weaning digestive disorders and productive of growing rabbits. Egypt. Poult. Sci., 34(1): 19-38.

Anderson, D.O., J.J. Hessen, Elser and J. Urabe,2005.Metabolicstoichiometry and the fate of excess carbon and nutrients in consumers. Am. Nat., 165: 1-15.Archetti, I., C .Titterelli, M. Cerioli, R. Brivio, G. Grilli and A. Lavazza, 2008: Serum chemistry and haematology values $\mathrm{m}$ in commercial rabbits: Preliminary data from industrial farms in northern Italy. Proceedings of 9th World Rabbit Congress, Verona, Italy: 1147-1152.
Archetti, I., C .Titterelli, M .Cerioli, R .Brivio, G .Grilli, A .Lavazza, 2008.Serum chemistry and haematologyvaluesin commercial rabbits: Preliminary data from industrial farms in northern Italy. Proceedings of 9th World RabbitCongress, Verona, Italy: 11471152.

Beshara, M.M.,M.A. Ragab, A.El.M.I. El Desoky, H.N.Fahim, A.M. El-Fhhat, A.M.Alazab and A.A. El-gamal, 2017. Effect of feed restriction with supplementation of probiotic on productive and economical performance in local growing rabbits. Egypt. Poult. Sci.Vol (37)(II):(407-431)(2017).

Birolo, M., A. Trocino, M .Tazzoli and G.Xiccato, 2017. Effect of feed restriction and feeding plans on performance, slaughter traits and body composition of growing rabbits. World Rabbit Sci. 25:113-122.

Cabaraux, J.F., M.Kerrour, C. VanEenaeme, I.Dufrasne, L.Istasse, J.L. Hornick, 2003. Different modes of food restriction and compensatory growth in double-muscled Belgian Blue bulls: plasma metabolites and hormones. Anim. Sci., 77: 205-214.

DalleZotte, A., 2000. Main factors influencing the rabbit carcass and meat quality. Proc. of the 7th World Rabbit Congress. Valencia, Spain. pp:1-32.

DalleZotte, A., H.Remigton, J. Ouhayoun, 2005. Effect of feed rationing during post-weaning growth on meat quality, muscle energy metabolism and fibre properties of Biceps femoris muscle in the rabbits. Meat Science, v.70, p.301-305, 2005.

Darina. C., E. T ${ }^{\circ}$ umova and Z.Volek, 2018 .The effect of limited feed intake on carcase yield and meat quality in early weaned rabbits .ITALIAN JOURNAL OF ANIMAL SCIENCE https:// doi.org/ 10.1080/1828051X.2018.1530961.

De Oliveira, M.C., R.P. Da Silva, L.S.Araujo, V.R. Da Silva, E.A.Bento and D.M.Da Silva, 2012.Effect of feed restriction on performance and feed digestibility in rabbits. Ital. J. Anim. Sci., 6: 765-767.

Di Meo, C., F. Bovera and S. Marono, 2007. Effect of feed restriction on performance and feed digestibility in rabbits. Italian Journal of Animal Science, v.6, p.765-767, 2007.

Duncan, D. B.,1955.Multiple range and multiple F-test, Biometrics, 11: 1-42. 38.

Ebeid, T., E.Tůmováand Z. Volek, 2012.Effects of a one week intensive feed restriction inthe growing rabbit: Part 1 - Performance and blood biochemical parameters. Proceedings 10th World Rabbit Congress - September 3 6, 2012-Sharm El- Sheikh -Egypt, pp 607 611.

El-Speiy, M.E., K.I. Kamel, A. E. Tag El-Din, A. E. Abd El-Hamid and A.M.E.ELKAmhawey,2015. Effect of feed restriction 
on productive performance, carcass yield, blood pictures and relative organ weights of growing rabbits. Egypt. Poult. Sci. Vol (35) (ii): (439-454)

Etim, N.A.N., M.E. Williams, U. Akpabio and E.A. Offiong, 2014. Haematological parameters and factors affecting their values. Agricultural Science 2: 37-47.

Fatma, A.E. andM.A.Hayam,2014 .Phaseolus vulgaris Straw as a Substitute for Clover Hay in Rabbit Diets with Prebiotic Supplementation and Feed Restriction Interaction: Influence on Nutrient Utilization, Caecal Activity, Carcass Yield and Blood Plasma Constituents. Global Veterinaria 13 (6): 1010-1021, 2014.

Foubert, C., J.Duperray, P.Boisot and A.Guyonvarch, 2008.Effect of feed restriction with or without free access to drinking water on performance of growing rabbits in healthy or epizootic rabbit enteropathy conditions. Proceedings 9th World Rabbit Congress, Verona, Italy, pp 667-671.

Gallois, M., T.Gidenne, L.FortunLamotheandI.L.Huerou-Luron, 2005.An early stimulation of solid feed intake slightly influences the morphological gut maturation in the rabbit. Reproduction Nutrition Development 45, 109-122.

Gidenne, T., A. Feugier, N. Jehl, P. Arveus, P. Boisot, C. Briens, E. Corrent, H. Fortune, S. Montessuy and S. Verdelhan, 2003. Unrationnementlimentairequantitatif postsevragepermet de réduire la fréquences des dierrhées, sans degradation importante des performances de croissance: résultatd'uneétude multi-site. In: Proc. 10émes Journ. Rech. Cunicole, 2003 November, Paris, France, 29-32.

Gidenne, T., S. Combes and L.Fortun-Lamothe, 2012.Feed intake limitation strategies for the growing rabbit: effect on feeding behaviour, welfare, performance, digestive physiology and health: a review. Animal, 6: 14071419.https://doi.org/10.1017/S175173111200 0389.

Gidenne,T., S. Combes, A. Feugier, N. Jehl, P. Arveux, P. Boisot, C. Briens and E. Corrent, 2009a. Feed restriction strategy in the growing rabbit. 2. Impact on digestive health, growth and carcass characteristics. Animal. 3:509-515.

Gidenne, T., S. Murr, A. Travel, E. Corrent, C. Foubert, K. Bebin, L. Mevel, G. Rebours and B. Renouf, 2009b. Effets du niveau de rationnement et du mode de distribution de l'alimentsur les performances et les troubles digestifspostsevrage du lapereau. Premiers re' sultatsd'une e' tudeconcerte'e du re' seau GEC. Cuniculture Magazine 36, 65-72.
Govaerts, T., G. Room and J. Buyse, 2000. Early and temporary quantitative food restriction of broiler chickens. 2. Effect on allometric growth and growth hormone secretion. British Poultry Science, v.41, p.355-362, 2000.

Guyton, A.C., J.E. Hall,2006.In: Textbook of Medical Physiology. $11^{\text {th }} \quad$ Edition. Philadelphia,Pa: Elsevier Saunders, 918-930.

Hewitt, C.D., D.J. Innes, J. Savory and M.R .Wills, 1989.Normal biochemical and haematological values in New Zealand White Rabbits.Clin Chem 35: 1777-1779.

Maertens, L., T.Gidenne,2016. Feed efficiency in rabbit production: nutritional, technicoeconomical and environmental aspects. In: Proc. 11th World Rabbit Congress Abstracts, 15-18 June, 2016, Quingdao, China, 131-151. Available at:http://pure.ilvo.vlaanderen.be/portal/files/4 411117/Maertens_Gidenne_WRC_2016.pdf. Accessed: July 2016.

Maertens, L., 2010. Feeding systems for intensive production.In: C. de Blas and J. Wiseman (Eds.) Nutrition of the Rabbit.2nd Edition. CAB International, Wallingford, Oxon, UK, 253- 266. https://doi.org/10.1079/9781845936693.02.

Martinec, M., H .Härtlová, D .Chodová, E .Tůmová, A .Fučíková, 2012.Selected haematological and biochemical indicators in different breed of rabbits. Acta Vet Brno 81: 371-375.

Mohammadalipour, R., H.R. Rahmani, R. Jahanian, A. Riasi, M. Mohammadalipour and N.Nili, 2017. Effect of early feed restriction on physiological responses, performance and ascites incidence in broiler chickens raised in normal or cold environment. Animal 11, 219-226.

Nilipous, A.H., 1998.Nunbres for successful poultry production world poulty, 14:26-28.

NRC, 1984. National Research Council. Nutrient Requirements of Rabbits. The 2nd Revised Edition. National Academy of Science. Washington. D.C. USA.

Oliveira M.C., R.P. Silva,L.S.Araújo,V.R. Silva,E.A. Bento and D.M. Silva 2012.Effect of feed restriction on performance of growing rabbits. R. Bras. Zootec.,41:1463-1467.

Özkan,C., A. Kaya and Y.Akgül,2012.Normal values of haematological and some biochemical parameters in serum and urine of New Zealand White rabbits. World Rabbit Sci 20: 253-259.

Rajman, M., M .Juráni, D .Lamošová, M .Máčajová, M .Sedlačková, L .Koštál, D. Ježová and P.Výboh, 2006.The effects of feed restriction on plasma biochemistry in growing meat type chickens (Gallus gallus). Comp Biochem Physiol A 145: 363-371. 
Renaville, R., C. Van Eenaeme, B.H.Breier, L.Vleurick, C.Bertozzi, N.Gengler, H.L.Hornick, I.Parmentier, L.Istasse, V.Haezebroeck, S. Massart and D. Portetelle, 2000.Feed restriction in young bulls alters the onset of puberty in relationship with plasma insulin-like growth factor-I (IGF-I) and IGF-binding proteins. Domest. Anim. Endocrin., $\quad 18$ : $165-176$. https://doi.org/10.1016/S0739 7240(99)00076-4.

Romero, C., S. Cuesta, J.R. Astillero, N. Nicodemus and C. De Blas, 2010.Effect of early feed restriction on performance and health status in growing rabbits slaughtered at $2 \mathrm{~kg}$ live-weight. World Rabbit Sci., 18: 211-218. https://doi. org/10.4995/wrs.2010.778.

SPSS., 2011. SPSS User's Guide Statistics. Ver. 20. Copyright SPSS Inc., USA.

Tůmova, E., M.Skoivan, V.Skoivanova, et al., 2002. Effect of early feed restriction on growth in broiler chickens, turkeys and rabbits. Czech Journal of Animal Science, v.47, p.418-428, 2002.
Tůmova, E., S.k. Rivanovaand M.S.k. Rivan, 2003. Effect of restricted feeding time and quantitative restriction in growing rabbits.Arch Gefl€ugelkunde.67:182-190.

Tůmová a, E., D.Chodová and Z.Volek, 2018.The effect of limited feed intake o carcase yield and meat quality in early weaned rabbits. ITALIAN JOURNAL OF ANIMAL SCIENCE. 26 July 2018.

Van Harten, S. and LA.Cardoso, 2010 .Feed restriction and genetic selection on the expression and activity of metabolism regulatory enzymes in rabbits. Animal 4: 1873-1883.

Yakubu, A., A.E.Salako, A.O. Ladokun, M.M.Adua, T.U.K. Bature, 2007.Effects of feed restriction on performance, carcass yield, relative organ weights and some linear body measurements of weaner rabbits. Pakistan J. Nutrition.6: 391- 396.

Young, D. S. and R. B. Friedman, 2001.Effects of disease on clinical laboratory tests (Vol. 1).Amer Assn for Clinical Chemistry.

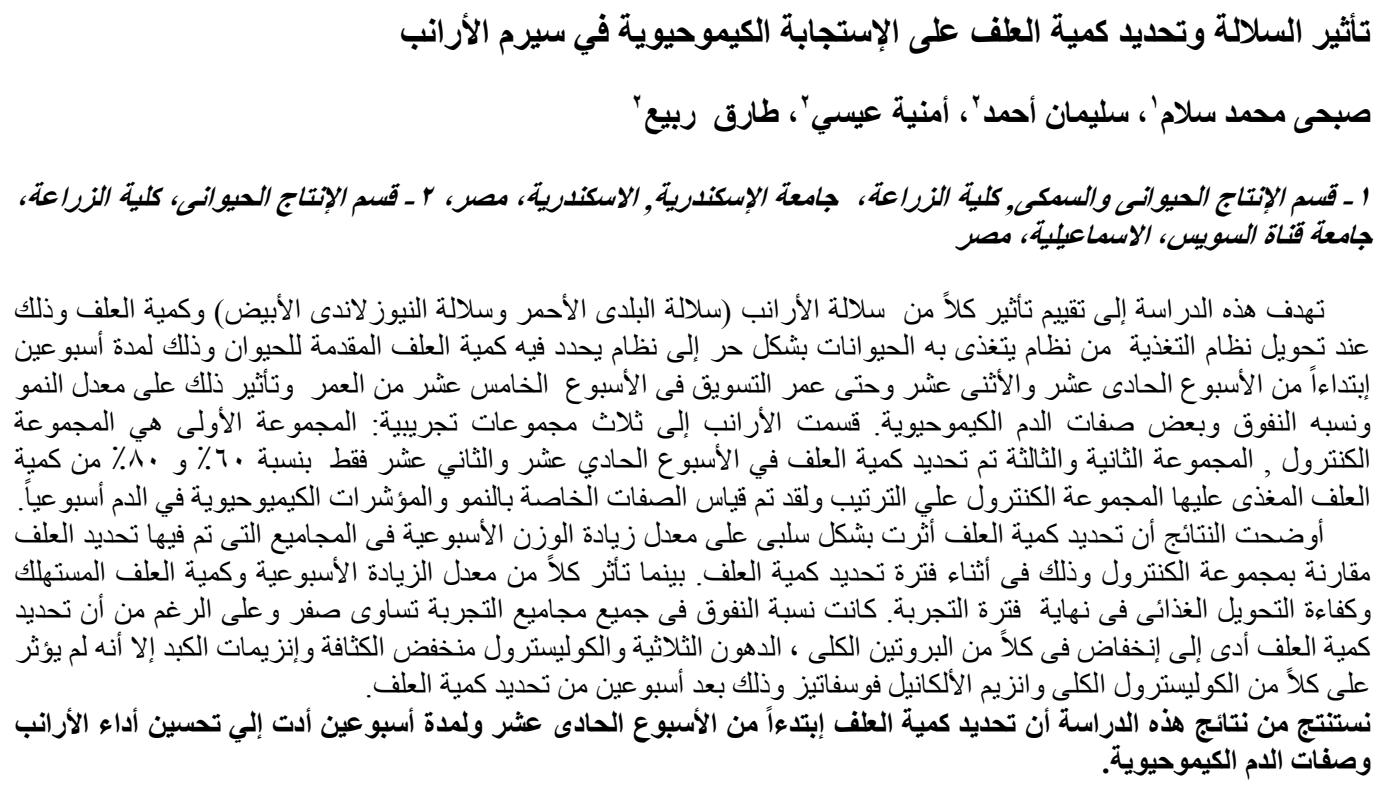

